\title{
A new method to study free transverse vibration of the human lumbar spine as segmental multi-layer Timoshenko and Euler-Bernoulli beams
}

\author{
H. Asgharzadeh Shirazi ${ }^{1}$, M. Fakher ${ }^{1}$, A. Asnafi ${ }^{2 *}$ and S. Hosseini Hashemi ${ }^{1,3}$
}

\begin{abstract}
Background: The aim of this study is to propose a method for studying the free transverse vibration of the human lumbar spine using Timoshenko and Euler-Bernoulli beam theories.

Methods: The cross section of the lumber spine is assumed to be uniform, and the material properties are different for the vertebral bodies, endplates, and intervertebral discs. To derive equations with biomedical approach, they were developed with $n$ segments of the lumbar spine including vertebrae, intervertebral discs, and endplates.

Results: Three first natural frequencies and mode shapes of system were computed and then validated with a finite element analyzer.

Conclusion: Due to good agreements between the results, it was concluded that the proposed method offered acceptable results; therefore, it can be applied to the entire spine from the neck region to the tailbone and pelvis ones.
\end{abstract}

Keywords: Human spine, Free transverse vibration, Tapered beam, Finite element method

\section{Background}

One of the most important characteristics of a structure is its free vibration behavior, which is especially important for identification and further response analysis of the structure. The human spine, as one of the most important organs in the body, plays a fundamental role in supporting the loads arising from both daily and specialized activities. These loads are mainly dynamic and periodic; therefore, a precise study on both the free and forced vibration of this structure may lead to a better understanding of the behavior of the system, especially in some activities, such as some professional exercises based on the rhythmic movements of the lumbar region. It may include some facts about the most effective exercises or dangerous movements that may be also applicable for many athletes. The results may also be used for better rehabilitation of injured persons.

\footnotetext{
* Correspondence: asnafi@shirazu.ac.ir

${ }^{2}$ Hydro-Aeronautical Research Center, Shiraz University, Shiraz 71348-13668, Iran

Full list of author information is available at the end of the article
}

Euler-Bernoulli and Timoshenko beam theories (Rao 2007) have been used in many researches (Han et al. 1999; Chaudhari and Maiti 2000; Lin and Chang 2005; Dong et al. 2005; Naguleswaran 2002) to study transverse vibration of thin and thick beams, respectively. These methods have been also used to model and solve multi-segment beams. For instance, the vibration of geometrically segmented beams with and without crack was studied by Chaudhari et al. (Chaudhari and Maiti 2000). They used Euler-Bernoulli theory and applied continuity as boundary conditions at the junction of the segments. The continuity conditions were in terms of displacement, slope, shear force, and bending moments. The free vibration analysis of a multi-span beam with an arbitrary number of flexible constraints was investigated by Lin et al. (Lin and Chang 2005). They used Timoshenko beam theory and considered the compatibility requirements on each constraint and, finally, determined eigen solutions of the entire system using a transfer matrix method. Dong et al. (Dong et al. 2005) investigated the 
free vibration of stepped laminated composite by Timoshenko beam theory. Naguleswaran (Naguleswaran 2002) evaluated the natural frequencies of a stepped Euler-Bernoulli beam with three step changes in cross section on elastic end supports.

From the viewpoint of anatomy, there are seven cervical vertebrae, twelve thoracic vertebrae, and five lumbar vertebrae in a human spine or vertebral column. Two adjacent vertebrae are separated from each other by superior endplate, intervertebral disc, and inferior endplate. Each part of the spine has a unique structure. The vertebra body is composed of the cancellous bone which is covered by a thin coating of the cortical bone. An intervertebral disc is also composed of an outer annulus which surrounds an inner gel-like center, i.e., the nucleus pulposus. If the cross section of the lumber spine is assumed to be almost uniform while the material properties are different for the vertebral bodies, endplates, and intervertebral discs, it can be considered as a beam-like structure whose natural frequencies can be evaluated using prescribed methods. Based on the actual structure of the human spine, it is essential to utilize the theories and models for segmental multi-layer beams. The previous reports in the literature have also employed such idealized model of the spine for decreasing the computational time and obtaining the exact analytical solution (Hussain et al. 2010; Asgharzadeh Shirazi and Ayatollahi 2014; Shahmohammadi et al. 2014). In this simplified case, adjacent vertebrae and their intervertebral discs in the lumbar region are ideally considered to be symmetric about their horizontal and vertical center planes. Although several studies have been performed on the free vibration analysis of the whole (Lan et al. 2013; Valentini and Pennestrì 2016) and lumbar (Wang et al. 2016; Guo et al. 2011; Fan and Guo 2017) spine via finite element method, limited information is available on the analytical free vibration examination of the lumbar spine. Due to the geometrical complexity, ordered or regular vibrational investigations of the human spine have not been fully accomplished; so, the research in this field is still widely open.

The main aim of this work is to develop an analytic approach to study the free transverse vibration of the human spine by using Timoshenko and Euler-Bernoulli beam theories. Furthermore, the lumbar region of the spine is selected for the current study because most degeneration and structural changes happened in this zone. In fact, this area is more vulnerable to damage than the other regions of the spine. Some defects such as disc degeneration and osteophyte formation are inevitable due to the natural procedures of aging and appeared as a result of changes in the structure and essence of the lumbar spine components. These phenomena would lead to biochemical and mechanical changes resulting from structural alterations especially in the nucleus pulposus and vertebral endplate. In the present work, the effects of these damages and defects, which occur over time and aging, have been theoretically and numerically investigated by changing the mechanical properties of the lumbar spine components. As also previously indicated, the cross section of the lumber spine is assumed to be uniform and the material properties are different for the vertebral bodies, endplates, and intervertebral discs.

\section{Methods}

\section{Problem formulation via Timoshenko and Euler-Bernoulli beam theories}

In this section, the governing equations of segmental beams were derived using Timoshenko and Euler-Bernoulli beam theories. Since vertebrae and intervertebral discs are composed of two different parts, as shown in Figs. 1 and 2, in and out subscripts are utilized to describe the inner and outer parts of each segment, respectively. It is noted that the endplate segments are homogenous and are all composed of one material.

\section{Timoshenko beam theory}

The bending moment and vertical force equilibrium equations including rotary inertia and shear deformation can be expressed in a differential form as (Timoshenko and Goodier 1970):

$$
\begin{aligned}
& \frac{d M}{d x}-Q=\int_{A_{\text {in }}} \rho_{\text {in }} \ddot{u}_{x} z d A+\int_{A_{\text {out }}} \rho_{\text {out }} \ddot{u}_{x} z d A \\
& \frac{d Q}{d x}=\int_{A_{\text {in }}} \rho_{\text {in }} \ddot{u}_{z} d A+\int_{A_{\text {out }}} \rho_{\text {out }} \ddot{u}_{z} d A
\end{aligned}
$$

where $\rho$ represents the mass density; $Q$ and $M$ are the stress resultants defined as:

$$
\begin{aligned}
& Q=\int_{A} \sigma_{x z} d A \\
& M=\int_{A} \sigma_{x x} z d A
\end{aligned}
$$

Stress-strain relations of the beam based on Hook's law can be written as: 


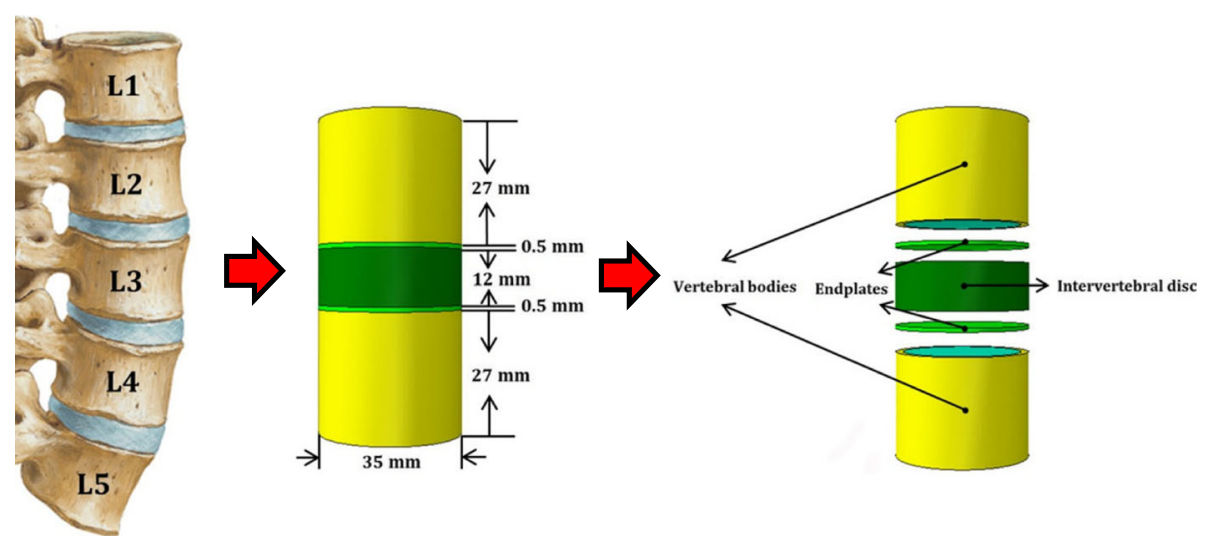

Fig. 1 Details of idealized disc-vertebral unit model of two adjacent vertebrae in order to develop to the whole lumbar spine (L1-L5)

$$
\begin{aligned}
& \left\{\begin{array}{c}
\sigma_{x x}=\left\{\begin{array}{cc}
E_{\mathrm{in}} \varepsilon_{x x} & 0 \leq r \leq R_{\mathrm{in}} \\
E_{\mathrm{out}} \varepsilon_{x x} & R_{\mathrm{in}} \leq r \leq R_{\mathrm{out}}
\end{array}\right. \\
\sigma_{x z}=\left\{\begin{array}{cc}
2 G_{\mathrm{in}} \varepsilon_{x z} & 0 \leq r \leq R_{\mathrm{in}} \\
2 G_{\mathrm{out}} \varepsilon_{x z} & R_{\mathrm{in}} \leq r \leq R_{\mathrm{out}}
\end{array}\right.
\end{array}\right. \\
& \text { where }\left\{\begin{array}{rlrl}
G_{\text {in }} & =\frac{E_{\text {in }}}{2\left(1+v_{\text {in }}\right)} & & 0 \leq r \leq R_{\text {in }} \\
G_{\text {out }}=\frac{E_{\text {out }}}{2\left(1+v_{\text {out }}\right)} & R_{\text {in }} \leq r \leq R_{\text {out }}
\end{array}\right.
\end{aligned}
$$

where $E$ is the elastic modulus, $v$ is the Poisson's ratio, and $G$ is the shear modulus of elasticity. The displacement fields based on Timoshenko beam theory is defined as:

$$
\left\{\begin{array}{l}
u_{x}=z \phi(x, t) \\
u_{z}=w(x, t)
\end{array}\right.
$$

where $\phi(x, t)$ and $w(x, t)$ are the rotation of cross section and vertical displacement of mid-plane at time $t$, respectively. So, the nonzero strains are given by:

$$
\left\{\begin{array}{l}
\varepsilon_{x x}=\frac{\partial u_{x}}{\partial x}=z \frac{\partial \phi(x, t)}{\partial x} \\
\varepsilon_{x z}=\frac{1}{2}\left(\frac{\partial u_{x}}{\partial z}+\frac{\partial u_{z}}{\partial x}\right)=\frac{1}{2}\left(\phi(x, t)+\frac{\partial w(x, t)}{\partial x}\right)
\end{array}\right.
$$

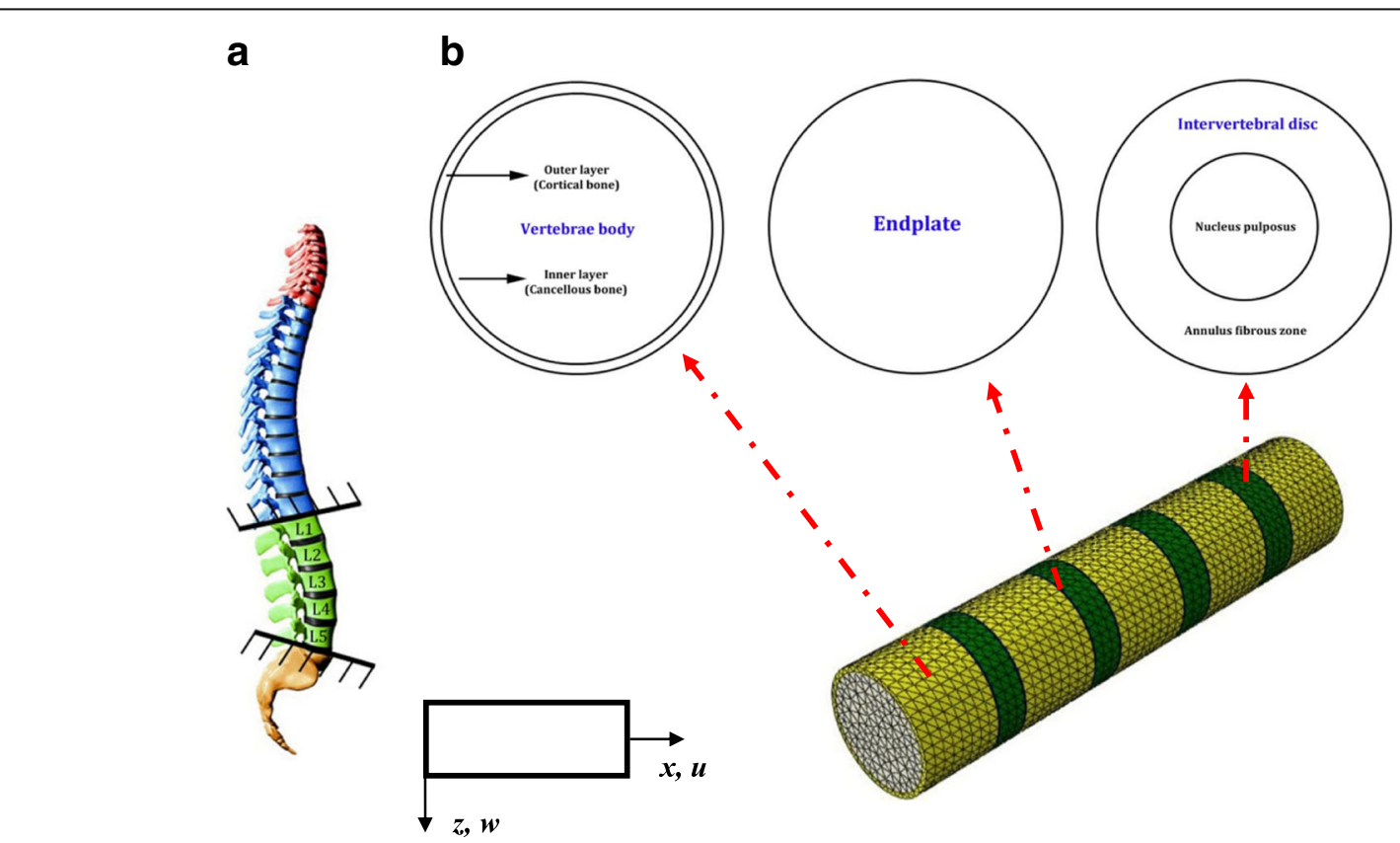

Fig. 2 a Clamped-clamped (CC) boundary conditions and $\mathbf{b}$ meshed geometry as well as details of the lumbar body components (cross section) for the whole lumbar spine model (L1-L5) 
By substituting Eq. (6) into Eq. (4), the stress fields as following equation would be in hand:

$$
\begin{aligned}
& \sigma_{z z}=0 \\
& \sigma_{x x}= \begin{cases}E_{\text {in }}\left(z \frac{\partial \phi}{\partial x}\right) & 0 \leq r \leq R_{\text {in }} \\
E_{\text {out }}\left(z \frac{\partial \phi}{\partial x}\right) & R_{\text {in }} \leq r \leq R_{\text {out }}\end{cases} \\
& \sigma_{x z}= \begin{cases}G_{\text {in }} k\left(\frac{\partial w}{\partial x}+\phi\right) & 0 \leq r \leq R_{\text {in }} \\
G_{\text {out }} k\left(\frac{\partial w}{\partial x}+\phi\right) & R_{\text {in }} \leq r \leq R_{\text {out }}\end{cases}
\end{aligned}
$$

in which $k$ denotes shear correction coefficient. By substituting Eq. (7) into Eqs. (3-a) and (3-b), the new versions of Eqs. (1) and (2) can be rewritten as:

$$
\begin{aligned}
& \left(\rho_{\text {in }} A_{\text {in }}+\rho_{\text {out }} A_{\text {out }}\right) \frac{\partial^{2} w}{\partial t^{2}}= \\
& \times\left(G_{\text {in }} A_{\text {in }}+G_{\text {out }} A_{\text {out }}\right) \\
& \times\left(\frac{\partial \phi}{\partial x}+\frac{\partial^{2} w}{\partial x^{2}}\right) \\
& \left(\rho_{\text {in }} I_{\text {in }}+\rho_{\text {out }} I_{\text {out }}\right) \frac{\partial^{2} \phi}{\partial t^{2}}+k\left(G_{\text {in }} A_{\text {in }}+G_{\text {out }} A_{\text {out }}\right) \\
& \times\left(\phi+\frac{\partial w}{\partial x}\right) \\
& =\left(E_{\text {in }} I_{\text {in }}+E_{\text {out }} I_{\text {out }}\right) \frac{\partial^{2} \phi}{\partial x^{2}}
\end{aligned}
$$

where $I$ is the moment of inertia of the cross section about its neutral axis. Also, the resultant moment and shear force are obtained as:

$$
\begin{aligned}
& M^{T}=\left(E_{\text {in }} I_{\text {in }}+E_{\text {out }} I_{\text {out }}\right) \frac{\partial \phi}{\partial x} \\
& Q^{T}=k\left(G_{\text {in }} A_{\text {in }}+G_{\text {out }} A_{\text {out }}\right)\left(\phi+\frac{\partial w}{\partial x}\right)
\end{aligned}
$$

\section{Euler-Bernoulli beam theory}

In Euler-Bernoulli beam theory, the rotational displacement of the cross section is corresponded to the slope of the vertical deflection while the shear deformation term is ignored, i.e., $\phi=-\partial w / \partial x$ (Timoshenko and Goodier 1970). Moreover, the rotational inertia effect is neglected. Therefore, the governing equation of the Euler-Bernoulli beam due to Eqs. (8) and (9) can be expressed as:

$$
\left(E_{\text {in }} I_{\text {in }}+E_{\text {out }} I_{\text {out }}\right) \frac{\partial^{4} w}{\partial x^{4}}=-\left(\rho_{\text {in }} A_{\text {in }}+\rho_{\text {out }} A_{\text {out }}\right) \frac{\partial^{2} w}{\partial t^{2}}
$$

The relative resultant moment and the shear force of the Euler-Bernoulli beam cross section are also presented as:

$$
\begin{aligned}
& M^{E}=-\left(E_{\text {in }} I_{\text {in }}+E_{\text {out }} I_{\text {out }}\right) \frac{\partial^{2} w}{\partial x^{2}} \\
& Q^{E}=-\left(E_{\text {in }} I_{\text {in }}+E_{\text {out }} I_{\text {out }}\right) \frac{\partial^{3} w}{\partial x^{3}}
\end{aligned}
$$

\section{FE simulation}

The geometry of the lumbar spine was developed based on anthropometric data, as shown in Fig. 1. In order to perform a numerical analysis, a geometry sample was modeled using finite element method for a comparative study. The finite element (FE) model was simulated using ABAQUS software (SIMULIA Corporation, Providence, RI, USA). Quadratic tetrahedral elements of type C3D10 were employed for meshing the model. There were 53,011 elements and 75,750 nodes in our model.

As shown in Figs. 1 and 2, there is a 3D model of L1-L5 segment including five vertebrae, eight endplates, and four intervertebral discs. The lumbar vertebra is modeled by a cancellous core surrounded by a 1.5 -mm-thick cortical layer. The ratio of the disc height to the height of the vertebral body is about $1: 2.25$ in the lumbar region. Cartilaginous endplates with $0.5 \mathrm{~mm}$ thickness were assumed at both superior and inferior surfaces of the intervertebral disc.

\section{Theory/calculation}

Free transverse vibration multi-layer segmental Timoshenko and Euler-Bernoulli beams

In this section, the analytic approach to study the free vibration of the lumbar spine as a muti-layer segmental beam is developed. As previously indicated, the beam has almost uniform cross section but is composed of the components with different geometry and mechanical properties. Figure 3 gives the reader an idea about the model of the multi-layer segmental beam to derive free vibrational equations in this work.

\section{Free transverse vibration analysis of Timoshenko beam}

In order to solve the governing equations of a Timoshenko beam, we consider both the slope and deflection profiles, $\phi(x, t)$ and $w(x, t)$ as the following form:

$$
\left\{\begin{array}{l}
w(x, t)=W(x) e^{i \lambda t} \\
\phi(x, t)=\Phi(x) e^{i \lambda t}
\end{array}\right.
$$

where $\lambda$ is the frequency of oscillation. By solving Eqs. (8) and (9), we have:

$$
\begin{aligned}
W_{j}(x)= & c_{1 j} \sin \xi_{1} x+c_{2 j} \cos \xi_{1} x+ \\
& c_{3 j} \sinh \xi_{2} x+c_{4 j} \cosh \xi_{2} x j=1,2,3, \ldots, n
\end{aligned}
$$




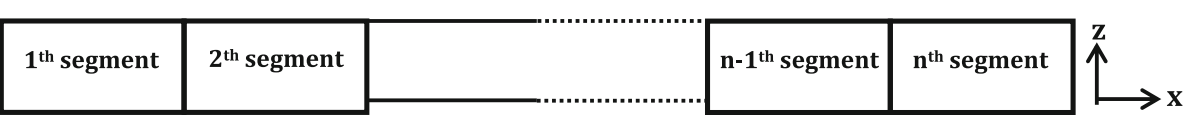

Fig. 3 Assumed tapered beam to derive free vibrational equations in this work

$$
\begin{aligned}
\Phi_{j}(x)= & k_{a} c_{2 j} \sin \xi_{1} x-k_{a} c_{1 j} \cos \xi_{1} x+ \\
& k_{b} c_{3 j} \cosh \xi_{2} x+k_{b} c_{4 j} \sinh \xi_{2} x j=1,2,3, \ldots, n
\end{aligned}
$$

In the above equation, $j$ relates to the free vibration solution of the $n$th segment of the beam. Also, the following parameters are defined as:

$$
\begin{aligned}
& \xi_{1}=\left(\frac{\eta_{1}+\sqrt{\eta_{1}^{2}-4 \eta_{2}}}{2}\right)^{\frac{1}{2}} \\
& \xi_{2}=\left(\frac{-\eta_{1}+\sqrt{\eta_{1}^{2}-4 \eta_{2}}}{2}\right)^{\frac{1}{2}} \\
& \eta_{1}=\frac{\Lambda_{1}}{\Lambda_{2}} ; \quad \eta_{2}=\frac{\Lambda_{3}}{\Lambda_{2}}
\end{aligned}
$$

where

$$
\begin{aligned}
& \left\{\begin{array}{l}
\Lambda_{1}=k_{1} k_{3}+k_{2} k_{4}-k_{3}^{2} \\
\Lambda_{2}=k_{2} k_{3} \\
\Lambda_{3}=k_{1} k_{4}
\end{array}\right. \\
& k_{1}=-\left(\rho_{\text {in }} I_{\text {in }}+\rho_{\text {out }} I_{\text {out }}\right) \lambda^{2}+k\left(G_{\text {in }} A_{\text {in }}+G_{\text {out }} A_{\text {out }}\right) ; \\
& k_{2}=-\left(E_{\text {in }} I_{\text {in }}+E_{\text {out }} I_{\text {out }}\right) ; k_{3}=k\left(G_{\text {in }} A_{\text {in }}+G_{\text {out }} A_{\text {out }}\right) ; \\
& \mathrm{k}_{4}=\left(\rho_{\text {in }} A_{\text {in }}+\rho_{\text {out }} A_{\text {out }}\right) \lambda^{2} ;
\end{aligned}
$$

Also $k_{a}$ and $k_{b}$ in Eq. (16-b) are:

$$
\left\{\begin{array}{l}
k_{a}=\frac{k_{3} \xi_{1}}{k_{1}-k_{2} \xi_{1}^{2}} \\
k_{b}=\frac{-k_{3} \xi_{2}}{k_{1}+k_{2} \xi_{2}^{2}}
\end{array}\right.
$$

To calculate the natural frequencies of Timoshenko beam, it is essential to satisfy the following boundary conditions:
Clamped - Clamped : $W_{1}(0)=\Phi_{1}(0)=0 ; W_{n}(L)=\Phi_{n}(L)=0$

Simply - Simply : $W_{1}(0)=M_{1}^{T}(0)=0 ; W_{n}(L)=M_{n}^{T}(L)=0$

Furthermore, following compatibility conditions should be established in the contact boundary between the different adjacent segments:

$$
\begin{aligned}
M_{j}^{T}\left(L_{j}^{*}\right) & =M_{j+1}^{T}\left(L_{j}^{*}\right) ; Q_{j}^{T}\left(L_{j}^{*}\right)=Q_{j+1}^{T}\left(L_{j}^{*}\right) ; j=1,2, \ldots, n-1 . \\
W_{j}\left(L_{j}^{*}\right) & =W_{j+1}\left(L_{j}^{*}\right) ; \Phi_{j}\left(L_{j}^{*}\right)=\Phi_{j+1}\left(L_{j}^{*}\right) ; j=1,2, \ldots, n-1 .
\end{aligned}
$$

where $L_{j}^{*}$ is the length of the contact place of $j$ th segment to $(j+1)$ th one. As it can be observed from above equations, there are $4 n$ equations and $4 n$ unknown coefficients that must be solved to have a nontrivial set of solution.

\section{Free transverse vibration analysis of Euler-Bernoulli beam}

In Euler-Bernoulli beam, to obtain the natural frequen-

\begin{tabular}{|c|c|c|c|c|c|c|}
\hline \multirow[t]{2}{*}{ Spinal components } & \multirow{2}{*}{$\begin{array}{l}\text { Density } \\
\left(10^{-6} \mathrm{~kg} /\right. \\
\left.\mathrm{mm}^{3}\right)\end{array}$} & Normal case & Case 1 & Case 2 & Case 3 & \multirow[t]{2}{*}{ Case 4} \\
\hline & & \multicolumn{4}{|c|}{ Elasticity modulus (MPa)-Poisson's ratio } & \\
\hline Cortical bone (Rohlmann et al. 2006; Zander et al. 2006) & 1.83 & $5000-0.30$ & $5000-0.30$ & $5000-0.30$ & $5000-0.30$ & $5000-0.30$ \\
\hline Cancellous bone (Rohlmann et al. 2006; Zander et al. 2006) & 1.00 & $500-0.25$ & $500-0.25$ & $500-0.25$ & $500-0.25$ & $500-0.25$ \\
\hline Endplate (Wang et al. 2016; Kurutz and Oroszváry 2010) & 1.83 & $100-0.40$ & $100-0.40$ & $100-0.40$ & $1000-0.30$ & $5000-0.30$ \\
\hline Nucleus pulpous (Asgharzadeh Shirazi and Ayatollahi 2014; Chen et al. 2008) & 1.36 & $10-0.49$ & $50-0.40$ & $100-0.30$ & $10-0.49$ & $10-0.49$ \\
\hline Annulus fibrous zone (Chen et al. 2001; Lee et al. 2000) & 1.20 & $175-0.30$ & $175-0.30$ & $175-0.30$ & $175-0.30$ & $175-0.30$ \\
\hline
\end{tabular}
cies, only the deflection profile is considered. In other words, it is assumed that the deflection profile, $w(x, t)$ has the following form:

$$
w(x, t)=W(x) e^{i \lambda t}
$$

By considering Eq. (22) and solving Eq. (12), we have:

$$
\begin{aligned}
& W_{j}(x)=c_{1 j} \sin \xi x+c_{2 j} \cos \xi x+c_{3 j} \sinh \xi x+c_{4 j} \cosh \xi x \\
& \quad j=1,2,3, \ldots, n
\end{aligned}
$$

where $j$ relates to the free vibration solution of the $n$th segment of the beam and $\xi_{1}$ and $\xi_{2}$ are also given by:

Table 1 Material properties of the model 
Table 2 Three first natural frequencies for all case studies

\begin{tabular}{|c|c|c|c|c|c|c|c|c|c|}
\hline \multirow[t]{2}{*}{ Cases } & \multicolumn{3}{|c|}{ Outcomes from equations (cycles/time) } & \multicolumn{3}{|c|}{ Outcomes from ABAQUS (cycles/time) } & \multicolumn{3}{|c|}{ Error (\%) } \\
\hline & N.F. 1 & N.F. 2 & N.F. 3 & N.F. 1 & N.F. 2 & N.F. 3 & N.F. 1 & N.F. 2 & N.F. 3 \\
\hline Normal case & 658.7 & 1423.5 & 2270.1 & 634.3 & 1329.0 & 2082.6 & 3.6 & 6.6 & 8.2 \\
\hline Case 1 & 687.4 & 1497.5 & 2402.5 & 686.5 & 1473.4 & 2351.6 & 0.1 & 1.6 & 2.1 \\
\hline Case 2 & 719.4 & 1580.4 & 2550.3 & 725.8 & 1581.1 & 2547.4 & 0.8 & 0.06 & 0.1 \\
\hline Case 3 & 685.0 & 1477.9 & 2355.3 & 667.3 & 1400.9 & 2199.6 & 2.6 & 5.2 & 6.6 \\
\hline Case 4 & 687.4 & 1482.9 & 2362.9 & 675.5 & 1418.4 & 2228.8 & 1.7 & 4.3 & 5.6 \\
\hline
\end{tabular}

$$
\xi=\left(\lambda \sqrt{\frac{\left(\rho_{\text {in }} A_{\text {in }}+\rho_{\text {out }} A_{\text {out }}\right)}{E_{\text {in }} I_{\text {in }}+E_{\text {out }} I_{\text {out }}}}\right)^{\frac{1}{2}} ;
$$

The following boundary conditions should be also satisfied to determine the natural frequencies of the EulerBernoulli beam:

Clamped - Clamped : $W_{1}(0)=\frac{\partial W_{1}(0)}{\partial x}=0 ; W_{n}(L)=\frac{\partial W_{n}}{\partial x}(L)=0$ Simply - Simply : $W_{1}(0)=M_{1}^{E}(0)=0 ; W_{n}(L)=M_{n}^{E}(L)=0$

In addition, following compatibility conditions should be considered in the contact boundaries between the adjacent segments:

$M_{j}^{E}\left(L_{j}^{*}\right)=M_{j+1}^{E}\left(L_{j}^{*}\right) ; Q_{j}^{E}\left(L_{j}^{*}\right)=Q_{j+1}^{E}\left(L_{j}^{*}\right) ; j=1,2, \ldots, n-1$.

$W_{j}\left(L_{j}^{*}\right)=W_{j+1}\left(L_{j}^{*}\right) ; \frac{\partial W_{j}\left(L_{j}^{*}\right)}{\partial x}=\frac{\partial W_{j+1}\left(L_{j}^{*}\right)}{\partial x} ; j=1,2, \ldots, n-1$.

Similar to what was proceeded for the Timoshenko beam, $4 n$ equations and $4 n$ unknown coefficients, it was found that in order to attain the nontrivial solution, the determinant of the coefficient matrix must be zero.

\section{Results}

Free transverse vibration analysis of the lumbar spine as a case study

The lumbar spine is composed of a set of discrete bony elements (vertebrae) connected by compliant structures such as endplates and intervertebral discs. This combination of unique elements, with different geometry and properties, gives the spine a great flexibility in the three-dimensional space to do even complex movements. The variation in material properties of spinal components owing to age, gender, and tissue degeneration may result in the changes in natural frequencies and modal modes of the structure. In this study, since the vertebrae and discs are more subject to damage and their characteristics change over time and aging, three first natural frequencies and modes of the lumbar spine were investigated for different mechanical properties of the endplates and the intervertebral discs. In fact, the changes in material properties of endplate and nucleus pulposus occur when they gradually lose their flexibilities and begin to tighten. In other words, the cartilaginous endplate converts slowly to a bony-like tissue and the nucleus loses its gel-like state

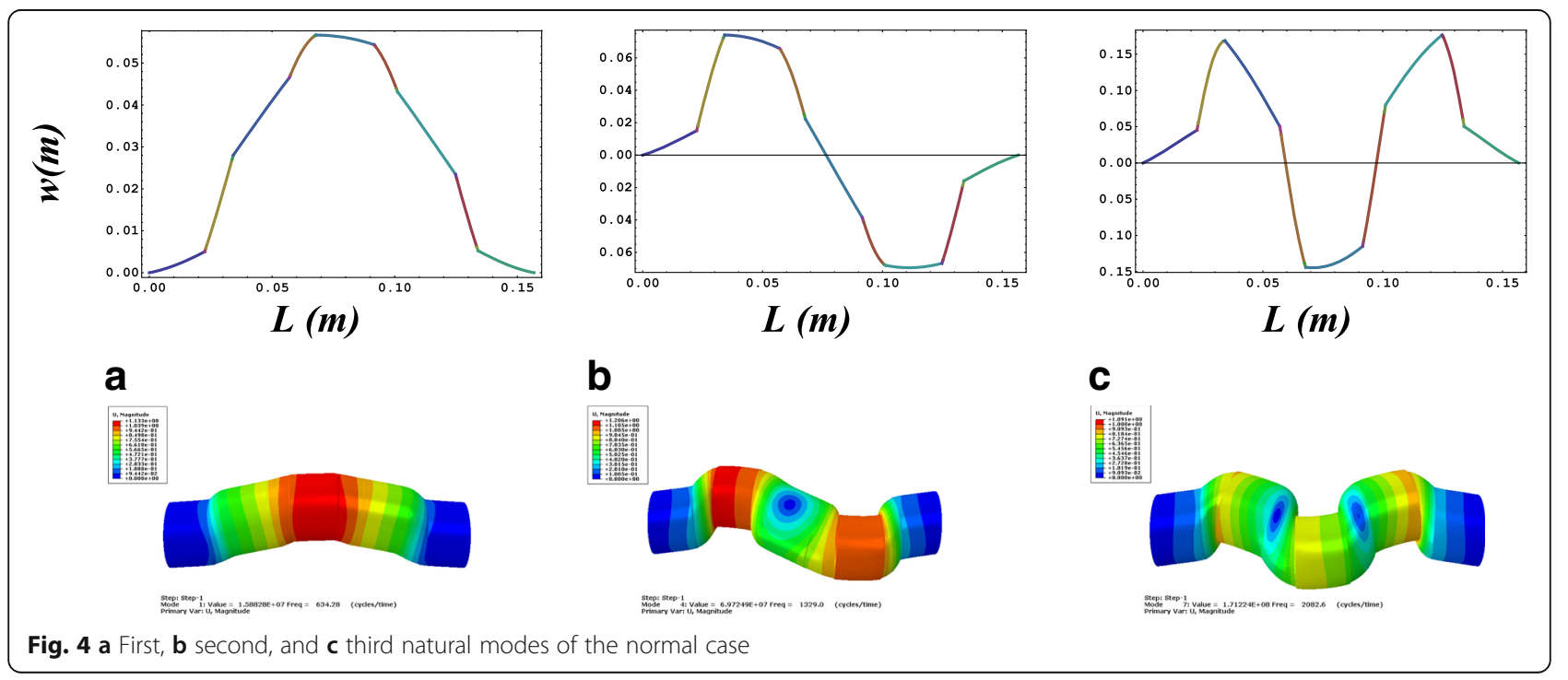




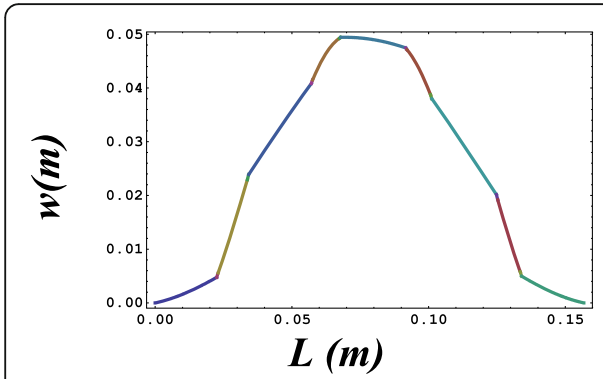

a

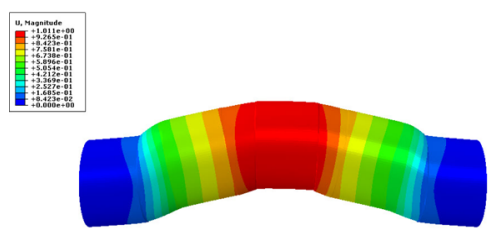

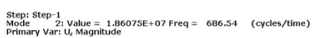

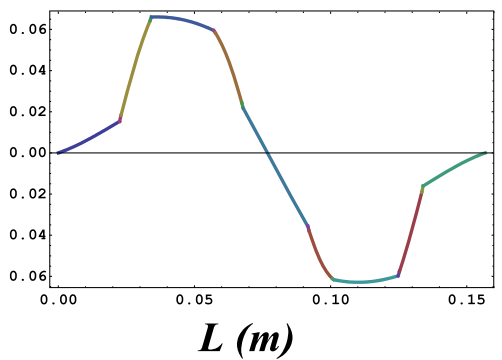

b

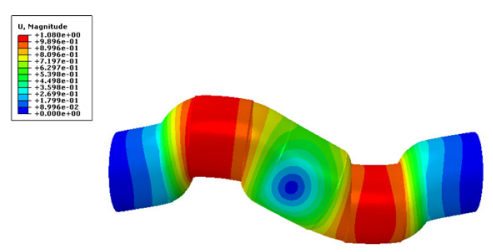

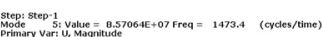

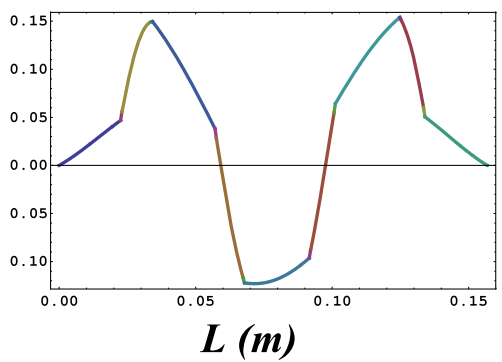

C

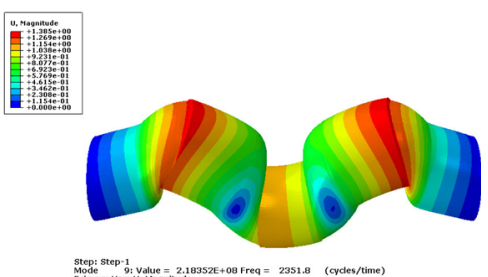

Fig. $\mathbf{5}$ a First, $\mathbf{b}$ second, and $\mathbf{c}$ third natural modes of case 1

and behaves like a solid. Table 1 indicates the various material properties of the lumbar spine components used in this work according to the previous literature. Moreover, clamped-clamped (CC) boundary conditions were considered because the lumbar spine was fixed between the thorax and the sacrum regions.

Table 2 lets one see the first natural frequencies obtained from the method presented in this article and those attained via FE solver. Figure 4 also demonstrates the first three natural mode shapes of the model for a normal body. It is to be noted that the results presented in Table 2 and Figs. 4, 5, 6, 7, and 8 are those obtained from the Timoshenko beam. In fact, both beams were modeled in this article and the results for both models were obtained but for the region that it was considered in this article i.e. L1-L5 (see Fig. 2), Timoshenko beam gave better results while for the whole spine which was not the goal of this paper, the other one worked better. The results accomplished via the method in this article and the finite element solver are drawn and compared to each other. Good agreements are seen for both the values of natural frequencies (Table 1) and the normal mode shapes (Fig. 4) that guarantee the correctness of the results.

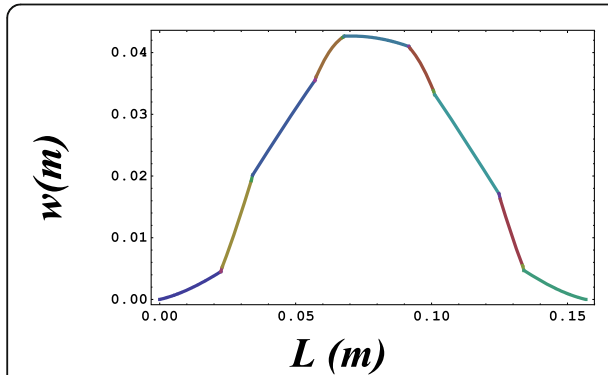

a

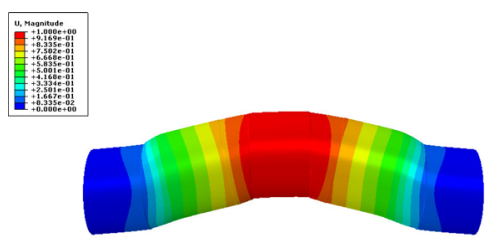

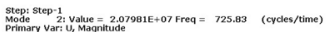

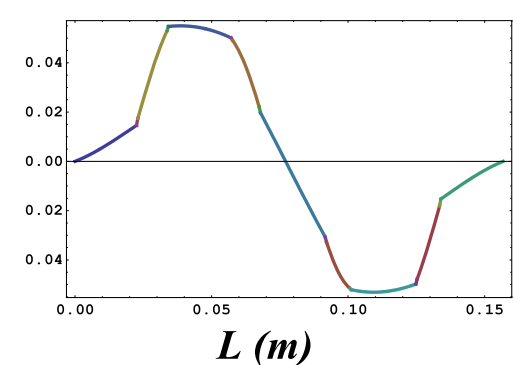

b

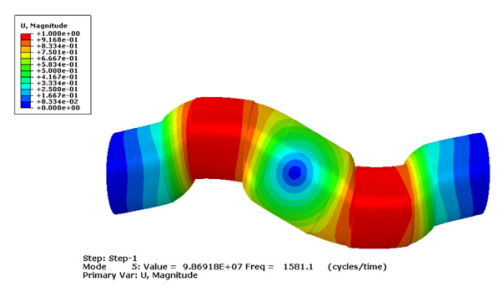

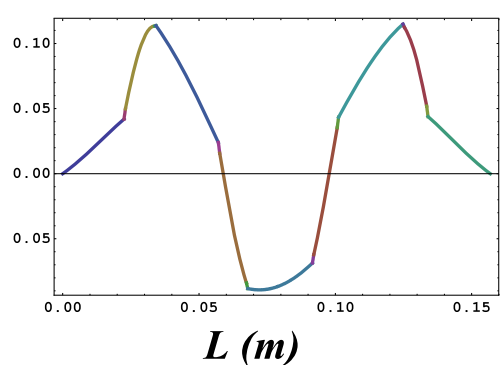

C

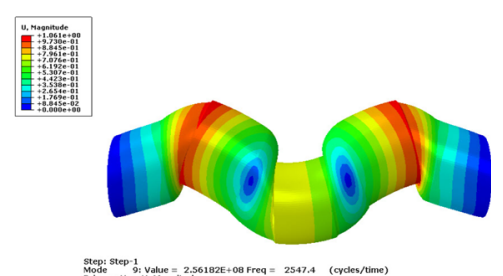

Fig. 6 a First, $\mathbf{b}$ second, and $\mathbf{c}$ third natural modes of case 2 


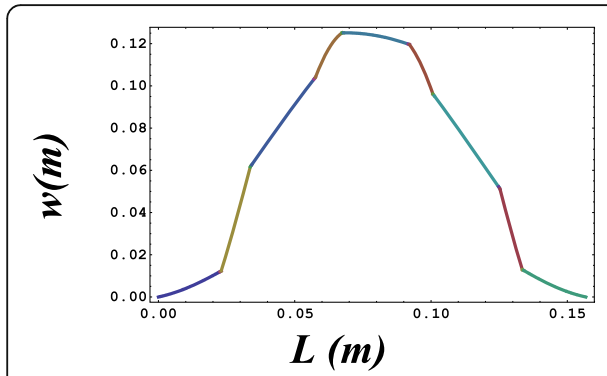

a

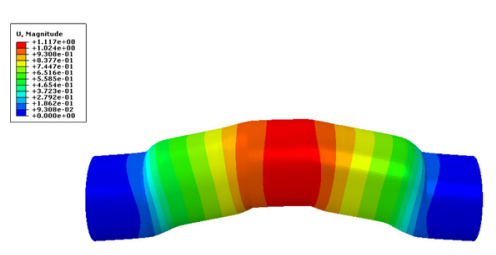

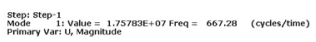

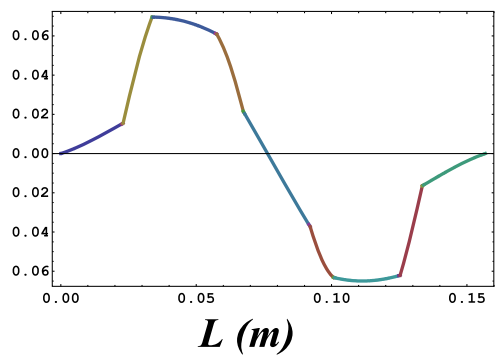

b

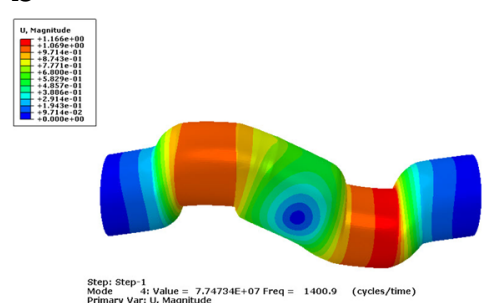

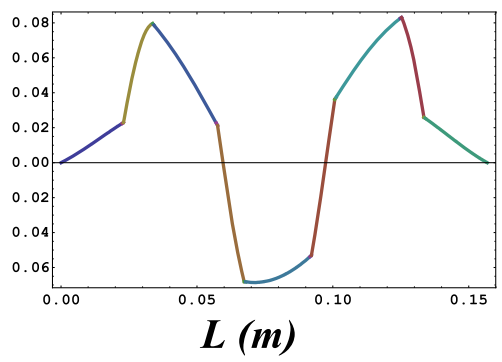

C

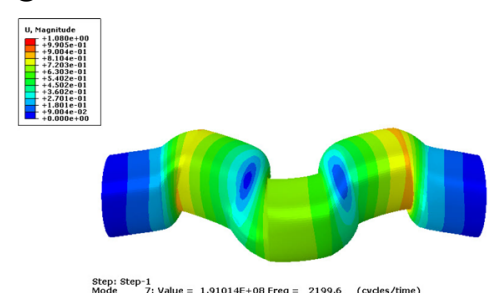

Fig. 7 a First, $\mathbf{b}$ second, and $\mathbf{c}$ third natural modes of case 3

\section{Discussion}

A validation study

As it can be seen in Table 2, all the analytical outcomes in all case studies are so close to those obtained via finite element simulator. This confirms the correctness of the method used and the results attained in this article.

\section{A discussion on the case studies}

As indicated previously, as we age, the flexibility of nucleus pulpous and endplates may decreases and they become stiffer than their normal situation. Figures 5 and 6 let someone see the effect of flexibility reduction of nucleus pulpous on natural frequencies. The modulus of elasticity increases five and ten times with respect to the normal case in cases 1 and 2 respectively. These result in an increase in natural frequencies (see Table 2). For better visual verification, the first three normal modes achieved from the analytic equations and the finite element solver are drawn and compared to each other.

Similar to previous cases, Figs. 7 and 8 illustrate the influence of flexibility reduction of endplates on natural frequencies. In cases 3 and 4, the endplate modulus of elasticity increases ten and 50 times with respect to the normal case respectively. Again, this causes an intensification in natural frequencies (see Table 2). Like previous cases, the first three normal modes obtained from the analytic

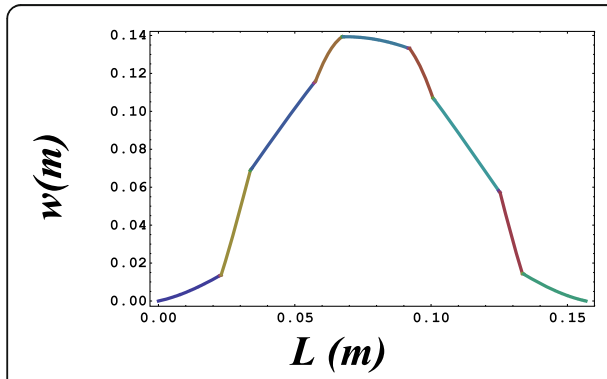

a

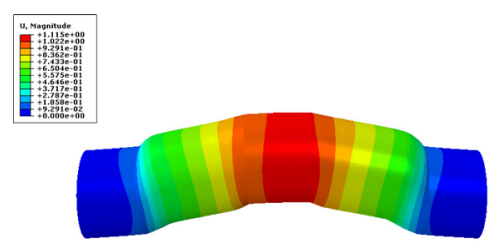

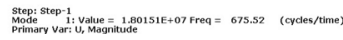

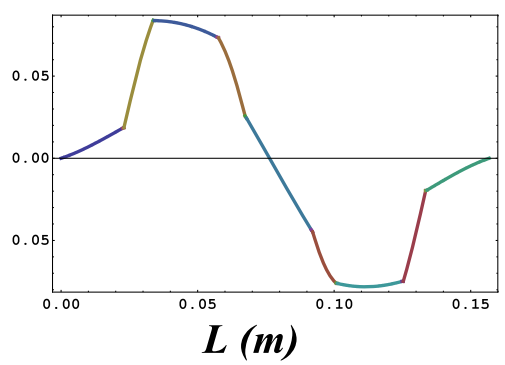

b

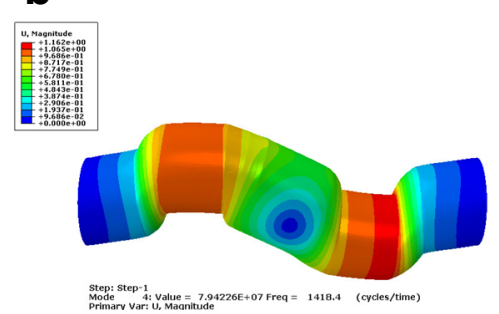

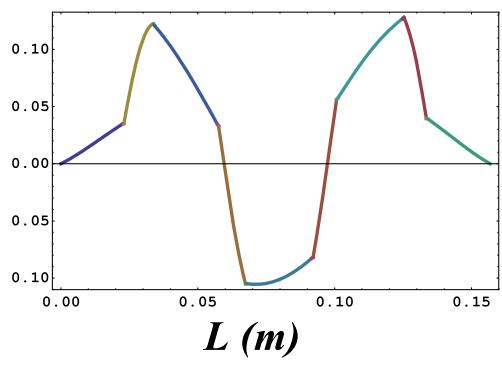

C

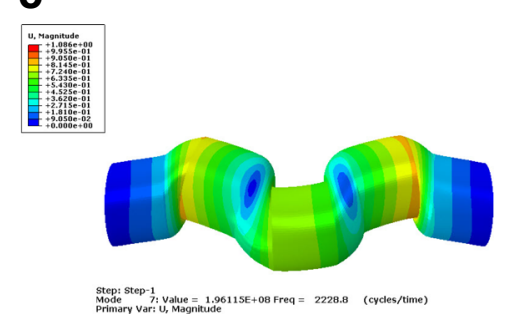

Fig. 8 a First, $\mathbf{b}$ second, and $\mathbf{c}$ third natural modes of case 4 
equations and the finite element solver are drawn and compared to each other for better visual confirmation.

\section{Conclusions}

Using Timoshenko and Euler-Bernoulli beam theories, as an analytic approach, the governing equations of transverse vibrations of both thick and thin beams were derived and applied for $n$ segments of the lumbar spine including vertebrae, intervertebral discs, and endplates. The developed system of equations was employed to obtain the natural frequencies and normal mode shapes of the model. The problem was similarly simulated in ABAQUS software, as the finite element analyzer, to validate the results attained from the analytical approach. The first three natural frequencies and mode shapes of the model for a normal body were calculated, drawn, and validated by finite element simulator outcomes. Good agreements are seen for both the values of natural frequencies and the normal mode shapes that guarantee the correctness of the results. The effect of flexibility reduction of both nucleus pulpous and endplates on the natural frequencies was investigated. It was shown that the natural frequencies become larger as the aging occur.

\begin{abstract}
Abbreviations
$L_{j}^{*}$ : The length of the contact place of $j$ th segment to $(j+1)$ th one; $\varphi(x, t)$ : The rotation of cross section of mid-plane at time $t ; \sigma_{i j}$ : The components of stress; $\varepsilon_{i j}$ : The components of strain; $A_{\text {in, }} A_{\text {out }}$ : The inner and outer surface areas; $E$ : The elastic modulus; $E_{\text {in }}, E_{\text {out }}$ The elastic modulus at inner and outer surfaces respectively; $G$ : The shear modulus of elasticity; $G_{\text {in }}, G_{\text {out }}$ : The shear modulus of elasticity at inner and outer surfaces respectively; I: The moment of inertia of the cross section about the neutral axis; $k$ : The shear correction coefficient; $M$ : The bending moment; $Q$ : The shear force; $r$ : Polar radius; $R_{\text {in, }}$ $R_{\text {out }}$ : The inner and outer radii; $u_{i}$ : The components of displacement; $w(x, t)$ : The deflection of the mid-plane at time $t ; x, y, z$ : Euclidian coordinates; $\lambda$ : The vibration frequency; $\rho$ : Mass density; $\rho_{\text {in }} \rho_{\text {out }}$ : Mass density at inner and outer surfaces; $u$ : The Poisson's ratio
\end{abstract}

\section{Authors' contributions}

HS surveyed the literature in this respect and prepared the history of the research and the first version of the article and also helped derive and solve the equations. MF derived and solved the equations and drew the figures in the corresponding softwares. AA gave the idea, interpreted the results, and wrote the final abstract, conclusion, and revision. SH investigated the correctness of the equations and text of the article. All authors read and approved the final manuscript.

\section{Authors' information}

$\mathrm{H}$ Asgharzadeh Shirazi is a PhD candidate of mechanical engineering at Iran University of Science and Technology, Theran, Iran.

M. Fakher is an alumni of Msc of mechanical engineering at Iran University of Science and Technology, Theran, Iran.

A Asnafi is an associate professor of mechanical engineering at Shiraz University, Shiraz Iran.

S Hosseini Hashemi is a professor of mechanical engineering at Iran University of Science and Technology, Theran, Iran.

\section{Competing interests}

The authors declare that they have no competing interests.

\section{Publisher's Note}

Springer Nature remains neutral with regard to jurisdictional claims in published maps and institutional affiliations.

\section{Author details}

${ }^{1}$ School of Mechanical Engineering, Iran University of Science and Technology, Narmak, Tehran 16846-13114, Iran. ${ }^{2}$ Hydro-Aeronautical Research Center, Shiraz University, Shiraz 71348-13668, Iran. ${ }^{3}$ Center of Excellence in Railway Transportation, Iran University of Science and Technology, Narmak, Tehran 16846-13114, Iran.

Received: 14 March 2018 Accepted: 2 July 2018

Published online: 13 July 2018

\section{References}

Asgharzadeh Shirazi, H, \& Ayatollahi, MR. (2014). Biomechanical analysis of functionally graded biomaterial disc in terms of motion and stress distribution in lumbar spine. International Journal of Engineering Science, 84, 62-78.

Chaudhari, TD, \& Maiti, SK. (2000). A study of vibration of geometrically segmented beams with and without crack. International Journal of Solids and Structures, 29, 761-779.

Chen, CS, Cheng, CK, Liu, CL, Lo, WH. (2001). Stress analysis of the disc adjacent to interbody fusion in lumbar spine. Medical Engineering \& Physics, 23, 485-493.

Chen, SH, Tai, CL, Lin, CY, Hsieh, PH, Chen, WP. (2008). Biomechanical comparison of a new stand-alone anterior lumbar interbody fusion cage with established fixation techniques - a three-dimensional finite element analysis. BMC Musculoskeletal Disorders, 9, 1-10.

Dong, XJ, Meng, G, Li, HG, Ye, L. (2005). Vibration analysis of a stepped laminated composite Timoshenko beam. Mechanics Research Communications, 32, 572-581.

Fan, W, \& Guo, LX. (2017). Influence of different frequencies of axial cyclic loading on time-domain vibration response of the lumbar spine: a finite element study. Computers in Biology and Medicine, 86, 75-81.

Guo, LX, Zhang, YM, Zhang, M. (2011). Finite element modeling and modal analysis of the human spine vibration configuration. IEEE Transactions on Biomedical Engineering, 58, 2987-2990.

Han, SM, Benaroya, H, Wei, T. (1999). Dynamics of transversely vibrating beams using four engineering theories. Journal of Sound and Vibration, 225, 935-988.

Hussain, M, Gay, RE, An, KN. (2010). Reduction in disk and fiber stresses by axial distraction is higher in cervical disk with fibers oriented toward the vertical rather than horizontal plane: a finite element model analysis. Journal of Manipulative and Physiological Therapeutics, 33, 252-260.

Kurutz, M, \& Oroszváry, L. (2010). Finite element analysis of weightbath hydrotraction treatment of degenerated lumbar spine segments in elastic phase. Journal of Biomechanics, 43, 433-441.

Lan, CC, Kuo, CS, Chen, CH, Hu, HT. (2013). Finite element analysis of biomechanical behavior of whole thoraco-lumbar spine with ligamentous effect. The Changhua Journal of Medicine, 11, 26-41.

Lee, CK, Kim, YE, Lee, CS, Hong, YM, Jung, JM, Goel, VK. (2000). Impact response of the intervertebral disc in a finite-element model. Spine, 25, 2431-2439.

Lin, HP, \& Chang, SC. (2005). Free vibration analysis of multi-span beams with intermediate flexible constraints. Journal of Sound and Vibration, 281, 155-169.

Naguleswaran, S. (2002). Vibration of an Euler-Bernoulli beam on elastic end supports and with up to three step changes in cross-section. International Journal of Mechanical Sciences, 44, 2541-2555.

Rao, SS (2007). Vibration of continuous systems. Hoboken: Wiley.

Rohlmann, A, Bauer, L, Zander, T, Bergmann, G, Wilke, HJ. (2006). Determination of trunk muscle forces for flexion and extension by using a validated finite element model of the lumbar spine and measured in vivo data. Journal of Biomechanics, 39, 981-989.

Shahmohammadi, M, Asgharzadeh Shirazi, H, Karimi, A, Navidbakhsh, M. (2014). Finite element simulation of an artificial intervertebral disc using fiber reinforced laminated composite model. Tissue \& Cell, 46, 299-303.

Timoshenko, S, \& Goodier, J (1970). Theory of elasticity. New York: McGraw-Hill.

Valentini, PP, \& Pennestrì, E. (2016). An improved three-dimensional multibody model of the human spine for vibrational investigations. Multibody System Dynamics, 36, 363-375.

Wang, S, Wang, L, Wang, Y, Du, C, Zhang, M, Fan, Y. (2016). Biomechanical analysis of combining head-down tilt traction with vibration for different grades of degeneration of the lumbar spine. Medical Engineering \& Physics, Article in press.

Zander, T, Rohlmann, A, Burra, NK, Bergmann, G. (2006). Effect of a posterior dynamic implant adjacent to a rigid spinal fixator. Clinical biomechanics, 21, 767-774. 